\title{
NGHIÊN CỬU NÂNG CAO HIỆU QUẢ CÔNG TÁC ĐO ĐẠC, XỬ LÝ SỐ LIỆU TRẮC ĐỊA TRONG THI CÔNG XÂY DỰíg NHÀ SIÊU CAO TÂNG Ở VIẸTT NAM
}

\author{
TRÀ̀ VIẾT TUÂN(1), DƯƠNG THÀNH TRUNG ${ }^{(1)}$, DIÊM CÔNG TRANG ${ }^{(2)}$ \\ (l)Truờng đại học Mỏ - Địa chất Hà Nội \\ (2) Viện KHCN Xây dụng
}

\section{Tóm tắt:}

Nội dung của bài báo trình bày kết quả nghiên cưu xây dụng thuật toán và chuoong trình máy tính nhằm nâng cao hiệu quả công tác đo đạc và xủ lý số liệu trắc địa phục vu thi công xây dụng công trình nhà siêu cao tầng. Kết quả đo đạc và xủ lý số liệu thực nghiệm nhằm đánh giá khả năng ưng dụng và độ chính xác đạt được của phần mềm superHBDV1.0 khi bố trí thi công xây dựng nhà cao tầng và sieu cao tầng ở Việt Nam.

\section{1. Đặt vấn đề}

Trong thi công xây dựng công trình nhà siêu cao tầng, để đảm bảo độ chính xác bố trí thi công xây dựng trong điều kiện công trình bị dao động do ảnh hưởng của các điều kiện ngoại cảnh và do bản thân tải trọng của công trình, chúng tôi đã đề nghị sử dụng công nghệ GNSS-RTK kết hợp với máy toàn đạc điện tử để bố trí chi tiết công trình [2]. Tuy nhiên khi sử dụng phương pháp này để bố trí chi tiết trên các sàn xây dựng cần phải xét đến yếu tố thời gian và tiến độ thi công xây dựng, đặc điểm và công nghệ thi công và các yêu cầu kỹ thuật cần thiết trong thi công xây dựng nhà siêu cao tầng trong điều kiện công trình luôn bị dao động do ảnh hưởng các yếu tố ngoại cảnh. Kết quả đo đạc và xử lý số liệu đo GNSS-RTK và toàn đạc điện tử phải xử lý tức thời ngay trên công truờng để có thể xác định được các yếu tố bố trí theo yêu cầu của đơn vị thiết kế và tư vấn giám sát công trình. Chính vì vậy mà cần phải nghiên cứu xây dựng thuật toán và chương trình máy tính thích hợp nhằm xác định các yếu tố bố trí công trình ngay trên các sàn thi công với độ chính xác tốt nhất trong thi công xây dựng các công trình nhà siêu cao tầng ở nước ta.

\section{Cơ sở lý thuyết và phương pháp nghiên cứu}

\subsection{Xây dụng thuật toán của chương trình}

\section{máy tính}

Theo kết quả nghiên cứu các giải pháp công nghệ bố trí chi tiết công trình nhà siêu cao tầng đã được trình bày trong các tài liệu [2], [3] Quy trình công nghệ công tác trắc địa bố trí thi công nhà siêu cao tầng bằng giải pháp sử dụng công nghệ GNSS-RTK kết hợp với máy toàn đạc điện tử bao gồm các nội dung sau đây:

2.1.1. Thành lập lưới khống chế thi công công trình trên mặt đất. Xác định toạ độ các điểm của lưới khống chế thi công trong hệ tọa độ thi công (sử dụng trị đo mặt đất bằng máy toàn đạc điện tử) và trong hệ VN-2000 bằng công nghệ đo GNSS tĩnh. Kết quả đo đạc lưới khống chế thi công trong 2 hệ toạ độ dùng để xác định:

+ Gốc hệ toạ độ địa diện chân trời [1], [3].

+ Điểm gốc của hệ toạ độ thi công công trình sử dụng làm điểm trạm base khi đo GNSS-RTK trong suốt qúa trình thi công xây dựng [2].

+ Dùng toạ độ các điểm song trùng trong hai hệ toạ độ để xác định các tham số tính chuyển Helmert dùng cho mục đích tính chuyển tọa độ các điểm đo GNSS-RTK về hệ toạ độ thi công công trình [1].[3].

2.1.2. Thu tín hiệu GNSS-RTK, truyền dẫn và ghép nối tín hiệu bằng sóng $3 \mathrm{G}$. Xử lý tín hiệu

Ngày nhận bài: 25/05/2020, ngày chuyển phản biện: 29/05/2020, ngày chấp nhận phản biện: 05/06/2020, ngày chấp nhận đăng: 08/06/2020 
thu GNSS-RTK loại bỏ những trị đo GNSS-RTK bất thường bằng thuật toán lọc Kalman [4].

2.1.3. Thuật toán tính chuyển toạ độ GNSS về hệ toạ độ thi công công trình [1].

2.1.4. Tính toạ độ điểm trạm máy toàn đạc điện tử " $T$ " bằng thuật toán giao hội nghịch góc - cạnh [2]

2.1.5. Tính các yếu tố bố trí theo phương pháp tọa độ cực.

\subsection{Thành lập chương trình máy tính}

Dựa vào các thuật toán nêu trên và ngôn ngữ lập trình $\mathrm{C} \#$, chúng tôi đã tiến hành thành lập chương trình máy tính có tên SuperHBDV1.0 để xử lý số liệu đo đạc phục vụ thi công xây dựng nhà siêu cao tầng ở Việt Nam. Phần mềm được thành lập chạy trên nền Windows, có giao diện đơn giản thuận tiện cho người sử dụng với khả năng tính toán nhanh, tức thời.

Giao diện của phần phần mềm SuperHBDV1.0 như (hình 1)

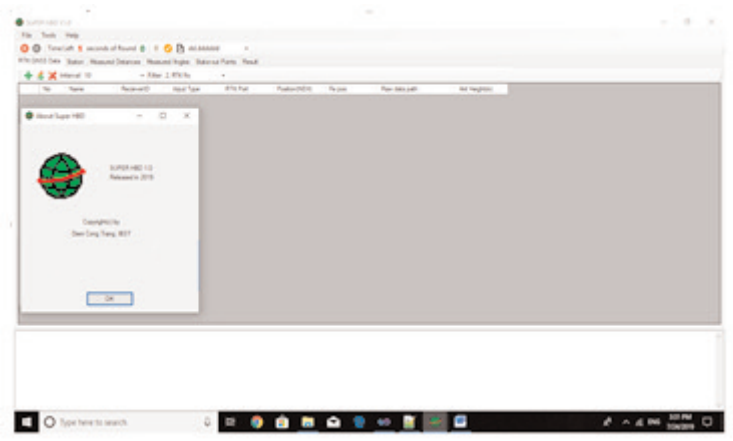

Trong phần mềm SuperHBD V1.0 có các modul chính sau đây:

- Modul cài đặt dữ liệu đo GNSS-RTK và các tham số khác.

- Cài đặt trạm máy toàn đạc điện tử và nhập số liệu đo giao hội góc cạnh.

- Cài đặt và nhập số liệu thiết kế công trình

- Modul hiển thị kết quả xử lý số liệu và độ chính xác đo đạc

\section{Kết quả đo đạc và tính toán thực nghiệm}

Để đánh gíá tính hiệu quả và độ chính xác của chương trình máy tính SuperHBD V1.0 chúng tôi đã tiến hành đo đạc thử nghiệm trên mô hình lưới khống chế thi công đã được thành lập kết nối với các điểm bố trí được xác định tọa độ trong hai hệ toạ độ tại khu vực Viện KHCN Xây dựng như (hình 2) [2].

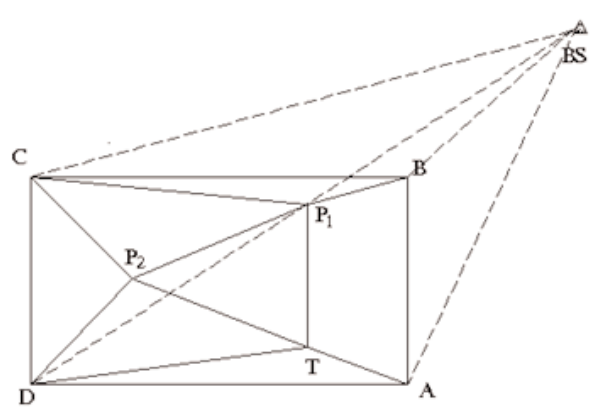

- Các điểm BS, A, B, C, D là các điểm của lưới khống chế thi công có toạ độ được xác định trong hệ toạ độ thi công và hệ VN- 2000 bằng công nghệ GNSS tĩnh

- Điểm $\mathrm{T}$ là điểm trạm máy, $\mathrm{P}_{1}$ và $\mathrm{P}_{2}$ là các điểm cần bố trí. Các điểm này đều có toạ độ trong hai hệ toạ độ nêu trên.

- Thiết bị sử dụng trong thực nghiệm là 03 máy thu GNSS-R8S và máy toàn đạc điện tử LaiKa TCR1201 có độ chính xác đo góc $\mathrm{m}_{\beta}= \pm$ 1 ", độ chính xác đo cạnh $\mathrm{m}_{\mathrm{S}}= \pm 1.5 \mathrm{~mm}$.

\subsection{Nội dung thục nghiệm}

\subsubsection{Thưc nghiệm 1}

Đầu tiên tiến hành nhập toạ độ của các điểm $\mathrm{A}, \mathrm{B}, \mathrm{C}, \mathrm{D}$ theo hai hệ toạ độ (toạ độ thi công và hệ VN-2000) vào phần mềm SuperHBD V1.0 để xác định các tham số tính chuyển Helmert.

- Phương án 1: Đặt một máy thu GNSS R8S tại điểm BS làm điểm trạm base. Hai máy thu GNSS R8S có gắn gương $360^{\circ}$ đặt tại các điểm $\mathrm{D}$ và $\mathrm{A}$ đã biết toạ độ. Máy toàn đạc điện tử đặt tại điểm $\mathrm{T}$, tiến hành đo góc và cạnh đến điểm $\mathrm{A}$ và $\mathrm{D}$ để xác định tọa độ điểm trạm máy $\mathrm{T}$. Kết quả đo như dòng (1) bảng 1 . 
Tiến hành thu tín hiệu GNSS-RTK tại 03 máy thu, truyền dẫn tín hiệu về phần mềm SuperHBD V1.0 bằng sóng $3 \mathrm{G}$. Sau khi lọc tín hiệu thu GNSS bằng bộ lọc Kalman $(\mathrm{KF})$ sẽ có toạ độ chính xác của các điểm $\mathrm{A}$ và $\mathrm{D}$. (Xem bảng 1)

Tại điểm $\mathrm{T}$ đo góc và cạnh đến điểm $\mathrm{P}_{1}$ và $\mathrm{P}_{2}$ (đã biết tọa độ) kết quả đo được $\mathrm{S}_{\mathrm{d}}$ và $\beta_{\mathrm{d}}$ như cột (2), (3) trên bảng 2 .

Trên phần mềm SuperHBD V1.0 Nhập số liệu giao hội góc và cạnh tại trạm máy $\mathrm{T}$ và số liệu tọa độ đã biết của hai điểm $\mathrm{P}_{1}$ và $\mathrm{P}_{2}$ (được coi là tọa độ thiết kế). Kết quả xử lý số liệu tại thời điểm $\mathrm{t}$ trên phần mềm cho các yếu tố bố trí góc bố trí $(\beta)_{\mathrm{TK}}$ và cạnh bố trí $(\mathrm{S})_{\mathrm{TK}}$ như cột (4), (5) trên bảng 2 . Độ lệch giữa kết quả đo kiểm tra so với số liệu bố trí tính toán trên phần mềm được nêu tại cột (7), (8) trên bảng 2. (Xem bảng 2)

$$
\text { Trong đó: } \begin{aligned}
\Delta_{\beta} & =\beta_{\mathrm{d}}-\beta_{\mathrm{TK}} \\
\mathrm{d}_{\mathrm{S}} & =\mathrm{S}_{\mathrm{d}}-\mathrm{S}_{\mathrm{TK}} \\
\mathrm{d}_{\beta} & =\left(\Delta_{\beta} / \rho\right) \mathrm{S}
\end{aligned}
$$

- Phương án 2: Đặt một máy thu GNSS R8S tại điểm BS làm điểm trạm base. Hai máy thu GNSS R8S có gắn gương $360^{\circ}$ đặt tại hai điểm bất kỳ $\left(\mathrm{A}_{1}, \mathrm{D}_{1}\right)$ không biết toạ độ toạ độ. Máy toàn đạc điện tử đặt tại điểm tuỳ ý (không biết toạ độ) tiến hành đo góc và cạnh đến điểm $\mathrm{A}_{1}$ và $\mathrm{D}_{1}$ để xác định toạ độ điểm trạm máy. Kết quả đo như dòng (2) bảng 1 .

Thực hiện lại các thao tác đo kiểm tra đến các điểm $\mathrm{P}_{1}$ và $\mathrm{P}_{2}$ như phương án 1 ta có kết quả đo kiểm tra được nêu tại các dòng (3), (4) của bảng 2

Nếu sử dụng toạ độ điểm trạm máy $\mathrm{T}$ xác định trên phần mểm SuperHBD V1.0 để tính tọa độ điểm $\mathrm{P}_{1}$ và $\mathrm{P}_{2}$ trên phần mềm để so sánh với toạ độ của các điểm này đã xác định trước ta có kết quả so sánh như bảng 3. (Xem bảng 3)

\subsubsection{Thưc nghiệm 2}

Để kiểm tra sai số trung phương tương hỗ bố trí các cạnh ra ngoài thực địa của phần mềm SuperHBD V1.0, chúng tôi đã sử dụng phần mềm để thiết kế một hình chữ nhật có kích thước $(15 \times 3)$ m và tính toán các yếu tố bố trí. Sử dụng

Bảng 1: Số liệu đo giao hội nghịch góc - cạnh tại trạm máy TĐĐT - T

\begin{tabular}{|c|c|c|c|c|c|c|c|c|}
\hline \multirow{2}{*}{\multicolumn{2}{|c|}{$\begin{array}{c}\text { Tên } \\
\text { điểm } \\
\text { KT }\end{array}$}} & \multicolumn{2}{|c|}{ Đo KT trên thực địa } & \multicolumn{2}{|c|}{ Số liệu theo thiết kế } & \multicolumn{3}{|c|}{ Độ lệch } \\
\hline & & Góc $\beta_{\mathrm{d}}$ & $\begin{array}{l}\mathrm{S}_{\tilde{a}} \\
(\mathrm{~m})\end{array}$ & Góc $\beta_{\mathrm{TK}}$ & $\begin{array}{l}\text { STK } \\
(\mathrm{m})\end{array}$ & $\begin{array}{l}\Delta_{\beta} \\
(")\end{array}$ & $\begin{array}{c}\mathrm{d}_{\mathrm{s}} \\
(\mathrm{mm})\end{array}$ & $\frac{\mathrm{d}_{\beta}}{(\mathrm{mm})}$ \\
\hline \multirow{2}{*}{\multicolumn{2}{|c|}{ (1) }} & (2) & (3) & (4) & (5) & (7) & (8) & (9) \\
\hline & & \multicolumn{6}{|c|}{ Phương án 1} & \\
\hline (1) & $\mathrm{P}_{1}$ & $305^{\circ} 48^{\prime} 58.2^{\prime \prime}$ & 17.330 & $305^{\circ} 49^{\prime} 24.0^{\prime \prime}$ & 17.332 & $-25.8^{\prime \prime}$ & -2 & 2.2 \\
\hline \multirow[t]{2}{*}{ (2) } & $\mathrm{P}_{2}$ & $221^{\circ} 35^{\prime} 55.8^{\prime \prime}$ & 17.547 & $221^{\circ} 36^{\prime} 17.5^{\prime \prime}$ & 17.544 & $-21.7^{\prime \prime}$ & +3 & 1.8 \\
\hline & & \multicolumn{6}{|c|}{ Phương án 2} & \\
\hline (3) & $P_{1}$ & $352^{\circ} 49^{\prime} 19.3^{\prime \prime}$ & 15.559 & $352^{\circ} 49^{\prime} 20.7^{\prime \prime}$ & 15.561 & $-0.4^{\prime \prime}$ & -2 & 0.03 \\
\hline (4) & $\mathrm{P}_{2}$ & $270^{\circ} 33^{\prime} 25.4^{\prime \prime}$ & 19.691 & $270^{\circ} 33^{\prime} 46.1^{\prime \prime}$ & 19.692 & $-20.7^{\prime \prime}$ & -1 & 1.9 \\
\hline
\end{tabular}

\begin{tabular}{|c|c|c|c|c|}
\hline \multicolumn{2}{|c|}{ TT } & Cạnh $\mathrm{S}_{1}(\mathrm{~m})$ & Cạnh $\mathrm{S}_{2}(\mathrm{~m})$ & Góc $\mathrm{GH}$ \\
\hline Phương án 1 & $(1)$ & 37.991 & 28.213 & $158^{\circ} 17^{\prime} 22^{\prime \prime} 3$ \\
\hline Phương án 2 & $(2)$ & 18.517 & 11.449 & $296^{\circ} 05^{\prime} 47.4^{\prime \prime}$ \\
\hline
\end{tabular}

Bảng 2: So sánh các yếu tố đo kiểm tra và các yếu tố bố trí tính trên SUPER HBD V1 
máy toàn đạc điện tử bố trí hình chữ nhật ở ngoài thực địa theo phương pháp toạ độ cực và đo kiểm tra lại tất cả các cạnh và hai đường chéo bằng thước thép. Kết quả đo kiểm tra cho như bảng 4. (Xem bảng 4)

\section{2. Đánh giá độ chính xác kết quả đo thụcc nghiệm đạt được}

3.2.1. Đánh giá độ chính xác bố trí điểm bằng công nghệ GNSS-RTK và phần mềm SuperHBD V1.0

Nếu coi kết quả đo kiểm tra trực tiếp các yếu tố bố trí điểm $\mathrm{P}_{1}$ và $\mathrm{P}_{2}$ có độ chính xác cao hơn kết quả thiết kế tính từ phần mềm, có thể đánh giá độ chính xác bố trí điểm chi tiết $\mathrm{m}_{\mathrm{P}}$ (theo các yếu tố bố trí tính từ phần mềm) theo công thức:

$$
\mathrm{m}_{\mathrm{p}}= \pm \sqrt{\frac{\sum \mathrm{d}^{2}}{\mathrm{n}}}
$$

Trong đó

$$
\begin{array}{r}
\mathrm{d}=\sqrt{\mathrm{m}_{\beta}^{2}+\mathrm{m}_{\mathrm{S}}^{2}} \\
\mathrm{~m}_{\beta}=\sqrt{\frac{\sum \mathrm{d}_{\beta}^{2}}{\mathrm{n}}} \\
\mathrm{m}_{\mathrm{S}}=\sqrt{\frac{\sum \mathrm{d}_{\mathrm{S}}^{2}}{\mathrm{n}}}
\end{array}
$$

Sử dụng các kết quả tính toán tại cột $(8),(9)$ trong bảng 2 để tính ta có: Sai số trong phương bố trí điểm chi tiết tính theo các yếu tố bố trí

$$
\mathrm{m}_{\mathrm{P}}= \pm 2.72 \mathrm{~mm}
$$

Bằng cách tính toán tương tự: khi sử dụng các số liệu đo tọa độ tại bảng 3 ta có: Sai số trong phương bố trí điểm chi tiết tính theo toạ độ

$$
\left(\mathrm{m}_{\mathrm{P}}\right)_{\mathrm{XY}}= \pm 2.23 \mathrm{~mm}
$$

3.2.2. Đánh giá độ chính xác bố trí cạnh bằng công nghệ GNSS-RTK và phần mềm SuperHBD V1.0

Sử dụng công thức (4) và các số liệu tính toán tại bảng 4 để tính, ta có: sai số trung phương bố trí chiều dài cạnh hay sai số trung phương tương hỗ giữa hai điểm:

$$
\mathrm{m}_{\mathrm{S}}= \pm 1.9 \mathrm{~mm}
$$

Từ kết quả đo đạc và tính toán thực nghiệm cho thấy: khi sử dụng công nghệ GNSS-RTK kết hợp với phần mềm SuperHBD V1.0 cho phép bố trí các điểm chi tiết trên công trình có độ chính xác đáp ứng được các yêu cầu về thời gian và các tiêu chuẩn kỹ thuật trong bố trí thi công xây dựng nhà siêu cao tầng [2].

Bảng 3: So sánh tọa độ đo kiểm tra và toạ độ thiết kế

\begin{tabular}{|c|c|c|c|c|c|c|c|}
\hline \multirow{2}{*}{$\begin{array}{c}\text { Số } \\
\text { thứ tự }\end{array}$} & \multirow{2}{*}{$\begin{array}{c}\text { Tên } \\
\text { điểm }\end{array}$} & \multicolumn{2}{|c|}{ Tọa độ bố trí } & \multicolumn{2}{|c|}{ Toạ độ thiết kế } & $\begin{array}{c}\text { Độ } \\
\text { lệch }\end{array}$ & $\begin{array}{c}\text { Độ } \\
\text { lệch }\end{array}$ \\
\cline { 3 - 8 } & & $\mathrm{X}_{\mathrm{bt}}(\mathrm{m})$ & $\mathrm{Y}_{\mathrm{bt}}(\mathrm{m})$ & $\mathrm{X}_{\mathrm{TK}}(\mathrm{m})$ & $\mathrm{Y}_{\mathrm{TK}}(\mathrm{m})$ & $\begin{array}{c}\mathrm{d}_{\mathrm{X}} \\
(\mathrm{mm})\end{array}$ & $\begin{array}{c}\mathrm{d}_{\mathrm{Y}} \\
(\mathrm{mm})\end{array}$ \\
\hline \multirow{2}{*}{$\begin{array}{c}\text { Phương } \\
\text { án 1 }\end{array}$} & $\left(\mathrm{P}_{1}\right)_{1}$ & 1021.669 & 496.720 & 1021.670 & 496.721 & -1 & -1 \\
\cline { 2 - 8 } & $\left(\mathrm{P}_{2}\right)_{1}$ & 1045.067 & 496.547 & 1045.065 & 496.548 & +2 & -1 \\
\hline \multirow{2}{*}{$\begin{array}{c}\text { Phương } \\
\text { án 2 }\end{array}$} & $\left(\mathrm{P}_{1}\right)_{\text {II }}$ & 1021.668 & 496.723 & 1021.670 & 496.721 & -2 & +2 \\
\cline { 2 - 8 } & $\left(\mathrm{P}_{2}\right)_{\text {II }}$ & 1045.067 & 496.547 & 1045.065 & 496.548 & +2 & -1 \\
\hline
\end{tabular}

Bảng 4: Kết quả đo kiểm tra chiều dài cạnh thực tế so với thiết kế

\begin{tabular}{|c|c|c|c|c|}
\hline TT & Tên cạnh & $\mathrm{S}_{\mathrm{tt}}(\mathrm{m})$ & $\mathrm{S}_{\mathrm{tk}}(\mathrm{m})$ & $\mathrm{d}_{\mathrm{s}}(\mathrm{mm})$ \\
\hline 1 & $1-2$ & 3.001 & 3.001 & 0.0 \\
\hline 2 & $2-3$ & 15.514 & 15.517 & -2.0 \\
\hline 3 & $3-4$ & 3.002 & 3.001 & 1.0 \\
\hline 4 & $4-1$ & 15.516 & 15.517 & -1.0 \\
\hline 5 & $2-4$ & 15.807 & 15.804 & 3.0 \\
\hline 6 & $1-3$ & 15.801 & 15.804 & -3.0 \\
\hline
\end{tabular}


Kết quả đo đạc thực nghiệm này cũng minh chứng cho tính hiệu quả, độ chính xác của giải pháp công nghệ và phần mềm thu và xử lý số liệu GNSS-RTK đã thành lập - SuperHBD V1.0

\section{Kết luận và kiến nghị}

Từ những kết quả nghiên cứu về lý thuyết, xây dựng thuật toán, thành lập phần mềm, đo đạc và tính toán thử nghiệm có thể rút ra một số kết luận sau:

- Giải pháp kỹ thuật sử dụng công nghệ GNSS- RTK kết hợp với phần mềm SuperHBD V1.0 cho phép bố trí các điểm chi tiết trên các sàn thi công nhà siêu cao tầng với độ chính xác đảm bảo được các yêu cầu kỹ thuật cần thiết mà tiêu chuẩn xây dựng đã đề ra. Giải pháp kỹ thuật này có thời gian đo đạc và xử lý số liệu rất nhanh (thời gian xử lý số liệu từ 3- 5 phút) điều này hoàn toàn phù hợp với đặc điểm thi công xây dựng các công trình nhà siêu cao tầng ở nước ta. Đó cũng chính là tính hiệu quả của nội dung nghiên cứu đã trình bày.

- Có thể mở rộng khả năng ứng dụng giải pháp kỹ thuật này trong công tác: tư vấn giám sát xây dựng, kiểm tra nghiệm thu và bố trí các công trình phức tạp, nhằm đem lại hiệu quả về kinh tế và kỹ thuật trong công tác trắc địa phục vụ thi công các công trình xây dựng ở Việt Nam. $\bigcirc$

\section{Tài liệu tham khảo}

[1]. Trần Viết Tuấn (2005), "Nghiên cứu phương pháp tính chuyển tọa độ các điểm đo GPS về hệ tọa độ thi công công trình", Tạp chí KHKT Mỏ - Địa chất số 11/7-2005, Hà Nội.

[2]. Trần Viết Tuấn, Diêm Công Trang (2019), "Nghiên cứu ứng dụng công nghệ GNSS-RTK trong thi công xây dựng nhà siêu cao tầng ở Việt Nam", Tạp chi khoa hoc đo đạc và bản đồ số 40, Hà Nội.

[3]. Trần Viết Tuấn, Diêm Công Trang (2019), "Nghiên cứu giải pháp kỹ thuật sử dụng công nghệ GNSS-RTK trong thi công xây dựng nhà siêu cao tầng ở Việt Nam", Tạp chí khoa học đo đạc và bản đồ số 42, Hà Nội.

[4]. Dương Thành Trung và nnk (2017), "Nghiên cưu xây dưng hệ thống định vị dẫn đuờng dựa trên việc tích hơp hệ thống vệ tinh dẫn đường toàn cầu (GNSS) và hẹ thống dẫn đường quán tính (INS) bằng các điều kiện ràng buộc giải tích và các thuật toán ước lượng tối uu“, Báo cáo tổng kết đề tài cấp bộ mã số B2015-02-22, Trường Đại học Mỏ -Địa chất, Hà Nội. $\bigcirc$

\section{Summary}

Research to improve the efficiency of geodetic data measurement and processing in the construction of super high-rise buildings in Vietnam

Tran Viet Tuan, Duong Thanh Trung

Hanoi University of Mining and Geology

Diem Cong Trang

Vietnam Institute for Building Science and Technology

The content of the article presents the results of researching and developing computer algorithms and programs to improve the efficiency of geodetic data measurement and processing for construction of super high-rise buildings. Results of measurement and processing of experimental data aim to evaluate the applicability and accuracy of SuperHBD V1.0 software when arranging construction of high-rise and super-high-rise buildings in Vietnam. $\bigcirc$ 